\title{
Avaliação multicriterial de desempenho e separação em aglomerados de fornecedores críticos de uma manufatura OKP
}

\author{
Everton Peter Santos da Rosa \\ Miguel Afonso Sellitto \\ Lia Weber Mendes \\ UNISINOS
}

\begin{abstract}
Resumo
O objetivo deste artigo é apresentar um estudo de caso de avaliação multicriterial do desempenho de dez fornecedores críticos de uma manufatura $O K P$, fabricação sob encomenda. Pela sua importância financeira e estratégica, selecionaram-se dez fornecedores para a avaliação. A metodologia de pesquisa constou de um grupo focado com os responsáveis pelas aquisições da empresa, que identificou os multicritérios de desempenho de fornecedores e, apoiado no método AHP, distribuiu importâncias relativas entre estes critérios, chegando às prioridades de desempenho. A estrutura de desempenho gerou um questionário multicriterial categórico, pelo qual os dez fornecedores foram avaliados por cinco compradores. Pelos resultados da avaliação, os fornecedores foram separados em dois, três e quatro aglomerados. A separação permite, na continuidade, a formulação de estratégias específicas para os aglomerados, o que pode ser uma contribuição para estudos em estratégia de aquisição em manufatura.
\end{abstract}

Palavras-chave

Avaliação de desempenho de fornecedores, aglomeração de fornecedores, AHP em compras, estratégia de aquisições, prioridades em aquisições.

\section{Performance multicriterial assessment and clusterization of critical suppliers in a one-of-a-kind manufacturer}

\begin{abstract}
This paper presents a case study of multicriterial performance assessment of ten critical supplier firms of a one-ofa-kind manufacture. The suppliers were selected according to financial and strategic importance. The methodology includes a focus group with purchasers of the company, which identified and assigned priorities, with the aid of AHP, to supplier performance multicriteria. The performance in purchasing structure achieved had yielded a categorical questionnaire used for five purchasers to evaluate the ten suppliers. According to the results, supplier firms were separated in two, three and four clusters, which allows, in continuity, the definition of specific purchasing strategy and this may be thought as a contribution to the study of this kind of strategy.
\end{abstract}

Key words

Supplier performance assessment, supplier clusterization, AHP in purchasing, purchasing strategy, priorities in purchasing. 


\section{INTRODUC̣̃̃O}

Um item relevante na disputa de mercado por empresas de fabricação de equipamentos é a capacidade de desenvolver vantagens competitivas em manufatura. Para Slack (2002), vantagem competitiva em manufatura significa: (i) fazer certo; (ii) fazer rápido; (iii) fazer pontualmente; (iv) poder mudar o que se faz; e (v) fazer com baixo custo. Merli (1994) fala em elementos de competitividade e objetivos estratégicos, ligados a custo, serviço, qualidade e inovação.

Uma alternativa para a busca de competitividade em manufatura é a criação de uma rede de empresas fornecedoras de itens e serviços. Segundo Krause, Scanell e Calantone (2000), empresas de manufatura têm enfocado esforços nas competências principais do negócio, contratando fora as atividades de fabricação e submontagens. Segundo Spekman e Hill apud Krause, Pagell e Curkovic (2001), a manufatura tem reconhecido a importância estratégica da função compras, ligando-a principalmente à estratégia de fabricação.

Um tipo de arranjo de manufatura é a fabricação sob encomenda, a one-of-a-kind product $(O K P)$. Cada unidade é projetada e construída segundo requisitos e especificações únicas. Hidrogeradores e transformadores de alta potência, como os produzidos na empresa estudada, são exemplos de $O K P$. Seu ciclo de produção é: (i) engenharia simplificada: faz a proposta; (ii) engenharia detalhada: estrutura o produto e define roteiros de fabricação; (iii) planejamento: cria o cronograma de compras e produção; (iv) compras: negocia com fornecedores e emite as ordens de compras; e (v) manufatura: recebe os itens comprados e monta o produto final. Mesmo com superposições de fases e engenharia simultânea, observam-se longos tempos até a entrega do produto, devidos principalmente a modificações no projeto e aos tempos de aquisição e recebimento de partes e subconjuntos. Na busca da competitividade em manufatura $O K P$, a velocidade na entrega se soma a outros critérios de desempenho, perseguidos em aquisições, tais como qualidade, preço e flexibilidade

O objetivo principal deste artigo é avaliar e classificar, segundo multicritérios de desempenho, dez fornecedores críticos de uma manufatura $O K P$. Os objetivos secundários são: (i) identificação dos fornecedores críticos; (ii) identificação e priorização dos critérios de desempenho; (iii) avaliação do desempenho dos fornecedores críticos segundo os critérios priorizados; e (iv) separação em aglomerados, segundo o desempenho avaliado. Remete-se à continuidade a definição das estratégias de compras aplicáveis aos aglomerados.

O método de trabalho começou pela escolha dos fornecedores a avaliar e seguiu com um grupo focado de compradores, que construiu a estrutura de desempenho e a priorizou pelo AHP (Analytic Hierarchy Process). Por questionário de escalas categóricas, gerado a partir da estrutura, os compradores avaliaram os fornecedores, que, segundo os resul- tados, foram separados em aglomerados. Fundamentações teóricas usadas não são revisadas (PEREIRA, 1999; MALHOTRA, 1999; HAIR et al., 1998; SAATY, 1991). O artigo está estruturado em: (i) introdução; (ii) revisão de temas que antecederam a pesquisa; (iii) metodologia e resultados; e (iv) discussão e continuidade.

A principal contribuição do artigo é a metodologia de separação de fornecedores em aglomerados, o que, associado a uma população de estratégias predefinidas, pode suportar a estratégia de aquisições. Classificado o fornecedor, emerge uma estrutura de objetivos e indicadores de desempenho correspondentes à estratégia pré-alocada ao aglomerado. Outras metodologias multivariadas surgem na literatura. Krause, Pagell e Curkovic (2001) selecionam fornecedores baseados em índices de desempenho e atribuição de importâncias relativas a prioridades padronizadas, tais como qualidade, custo, entrega e flexibilidade, e Chen, Paulraj e Lado (2004) usam equações estruturais para testar hipóteses que relacionam capacidades dinâmicas e objetivos padronizados de compras.

\section{DESEMPENHO COMPETITIVO EM MANUFATURA E SUPRIMENTOS}

O papel da estratégia de manufatura é alcançar os objetivos impostos pela estratégia de negócios, criando e mantendo uma vantagem competitiva baseada nas práticas de manufatura. Parafraseando Skinner (1969), a competição baseada em manufatura é o elo que conecta a estratégia de operação à estratégia de negócios.

Para Davis, Aquilano e Chase (2001), mudou o papel estratégico da manufatura. No passado, seu objetivo de competição era a redução de custos. Recentemente, a competição também tem objetivado a diferenciação de produto e o aumento de valor agregado ao cliente por serviços associados ao produto.

Autores identificaram objetivos de competição em manufatura. Slack (2002) aponta: (i) qualidade; (ii) velocidade; (iii) confiabilidade; (iv) flexibilidade; e (v) custo. Contador (1996) aponta: (i) preço; (ii) produto; (iii) prazo até o uso; (iv) assistência e serviços; e (v) imagem associada ao produto. Skinner (1976, apud DAVIS; AQUILANO; CHASE, 2001) aponta: (i) custo; (ii) qualidade; (iii) entrega; e (iv) flexibilidade. Davis, Aquilano e Chase (2001) acrescentam uma quinta prioridade, serviços associados ao uso do produto. Paiva, Carvalho e Fensterseifer (2004) falam em: (i) custo; (ii) qualidade; (iii) flexibilidade; (iv) desempenho de entrega; e (v) inovatividade. Sellitto e Walter (2006) apresentam um caso em manufatura eletrônica em que os objetivos de competição são: (i) tecnologia; (ii) qualidade; (iii) comunicação; (iv) serviços; (v) entrega; e (vi) custo.

Segundo Slack (2002), os objetivos de competitividade podem não ter a mesma importância relativa, já que esta 
depende do posicionamento da empresa focal na indústria. Sellitto e Walter (2006) acrescentam que a importância relativa também pode variar no tempo, devido a modificações tecnológicas e da entrada e saída de atores na competição.

Uma alternativa para o atendimento de múltiplos requisitos de competição em manufatura é a externalização de atividades. Andrade e Furtado (2005) caracterizam-na como a transferência da produção a empresas externas. Neste caso, não cabe à manufatura a gestão do processo de fabricação, mas a gestão de seus resultados. Segundo Krause, Pagell e Curkovic (2001), para externalizar itens, a manufatura deve medir o desempenho dos fornecedores. Quando este não satisfaz,

a manufatura pode retomar a produção do item, mudar de fornecedor ou ajudá-lo a melhorar o desempenho (PIRES, 2004). Krause, Scanell e Calantone (2000) citam estratégias cujos objetivos condicionam o desempenho de fornecedores: (i) pressão competitiva e concorrência; (ii) avaliação de fornecedores e realimentação de informações para a correção do desempenho; e (iii) aumento de volume de negócios condicionados ao desempenho.

A prática da externalização faz surgir uma rede de fornecedores de apoio à função compras.

Segundo Ellram e Carr (1994), a função compras escolhe e desenvolve os fornecedores que suportarão a estratégia de produção e o posicionamento competitivo do negócio. Segundo Arnold (1999), os objetivos da função compras são: (i) especificar as aquisições requeridas pela manufatura, em termos de quantidades, exigências funcionais e preços; (ii) comprar segundo estas especificações; (iii) garantir as entregas; e (iv) manter boas relações com potenciais fornecedores. Para atingir estes objetivos, é necessário: (i) especificar as necessidades de compras; (ii) selecionar os fornecedores potenciais e desenvolvê-los; (iii) negociar termos e acordos de entregas; (vi) administrar os ciclos de pedidos; (v) mensurar o desempenho de fornecedores; (vi) classificar, certificar e gerenciar os fornecedores segundo as classes; e sempre (vii) cooperar com fornecedores.

Para desenvolver fornecedores, testam-se empresas com potencial em projeto e processo, através de teste de amostras e lotes-piloto, em ações de avaliação de amostras, auditorias em processos de fabricação, compartilhamento de informações, tais como previsão de demandas, e suporte técnico a ações corretivas (ARNOLD, 1999). Os fatores de desempenho a serem avaliados podem ser qualificadores, que permitem que o fornecedor entre na disputa, e ganhadores de pedidos, que, dentre os qualificados, definem o vencedor (HILL, 1985).
Slack (2002) afirma que uma operação de manufatura é um elo em uma rede complexa, tal como uma cadeia de suprimentos. Para Quinn et al. apud Krause, Scanell e Calantone (2000), a habilidade de coordenar atividades internas com suprimentos externos pode ser uma arma de competição em manufatura. Para Christopher (2002), uma cadeia de suprimentos é uma rede de organizações, com ligações nos dois sentidos entre os diferentes processos e atividades que produzem bens e serviços entregues ao consumidor final. A cadeia inicia-se com a matéria-prima, passa pela entrega ao cliente final e termina no retorno de resíduos e de produtos que eventualmente não foram consumidos ou vendidos. 
intermediária entre as classes $\mathrm{A}$ e $\mathrm{C}$; e (iii) classe $\mathrm{C}$ : grupo de itens menos importantes que justificam pouca atenção ou uma estratégia comum simplificada. Brown (1965) observa que a curva $\mathrm{ABC}$ pode ser modelada pela distribuição lognormal, o que pode ser útil, caso se deseje realizar um cálculo exato para os limites entre as classes.

A curva $\mathrm{ABC}$ da manufatura $O K P$ estudada surge na Figura 1 . Os fornecedores de classe A fornecem $10 \%$ dos itens, que perfazem cerca de $70 \%$ do valor de compras da empresa. É nesta classe que os dez fornecedores críticos deste estudo serão identificados.

Outro instrumento de classificação de fornecedores é a matriz de importância estratégica e criticidade (IEC), apresentada em Dornier et al. (2000), que categoriza as aquisições em duas dimensões: o valor estratégico do componente e a criticidade para o produto final.

O julgamento sobre o valor estratégico do componente inclui a sua complexidade tecnológica, a natureza proprietária de sua tecnologia e a escassez de alternativas. O julgamento sobre a criticidade inclui o valor percentual e o grau de dependência do produto final em relação ao componente. O julgamento gera quatro classes: inovações, propriedades, commodities e utilidades. Inovações são itens que necessitam tecnologia sofisticada, mas não são essenciais para o produto final; propriedades são os produtos básicos (core products) da empresa; commodities envolvem baixa tecnologia, baixa diferenciação e participação mínima no produto final; e utilidades são itens críticos para o produto final, mas de tecnologia baixa ou plenamente acessível (DORNIER et al., 2000).

O julgamento pode ser representado graficamente, como na Figura 2.

Exemplos das classes de materiais na manufatura estudada são: (i) dispositivos eletrônicos de sinalização e proteção são inovações; (ii) parafusos e tintas são commodities; (iii) comutação de tensão e sustentação capacitiva são propriedades; e (iv) vernizes e bobinas são utilidades.

Uma alternativa de classificação de materiais surge em Klippell e Antunes Jr. (2002), que citam Carter (1999) e Grieco (1995). Os autores analisam dimensões de risco e valor. A dimensão de risco considera: (i) o poder de barganha de fornecedores; (ii) o potencial de substituição do fornecedor; (iii) a rivalidade no setor; e (iv) a existência de barreiras à entrada de fornecedores. A dimensão de valor considera a influência do item em dimensões competitivas do produto, tais como: custo, qualidade, entrega ou tecnologia. Dos julgamentos podem resultar quatro classes: (i) componentes não-críticos (baixo risco no suprimento e baixa influência em competição); (ii) componentes estratégicos (alto risco e alta influência); (iii) componentes de risco (alto risco e baixa influência); e (iv) componentes de competição (baixo risco e alta influência). A classificação também pode ser apresentada de forma gráfica, como a matriz anterior.

Uma terceira forma matricial de classificar fornecedores surge em Pires (2004), que cita Handfield et al. (2000). A classificação ocorre após o julgamento que estrategistas fa-

Figura 1: A curva ABC da manufatura estudada.

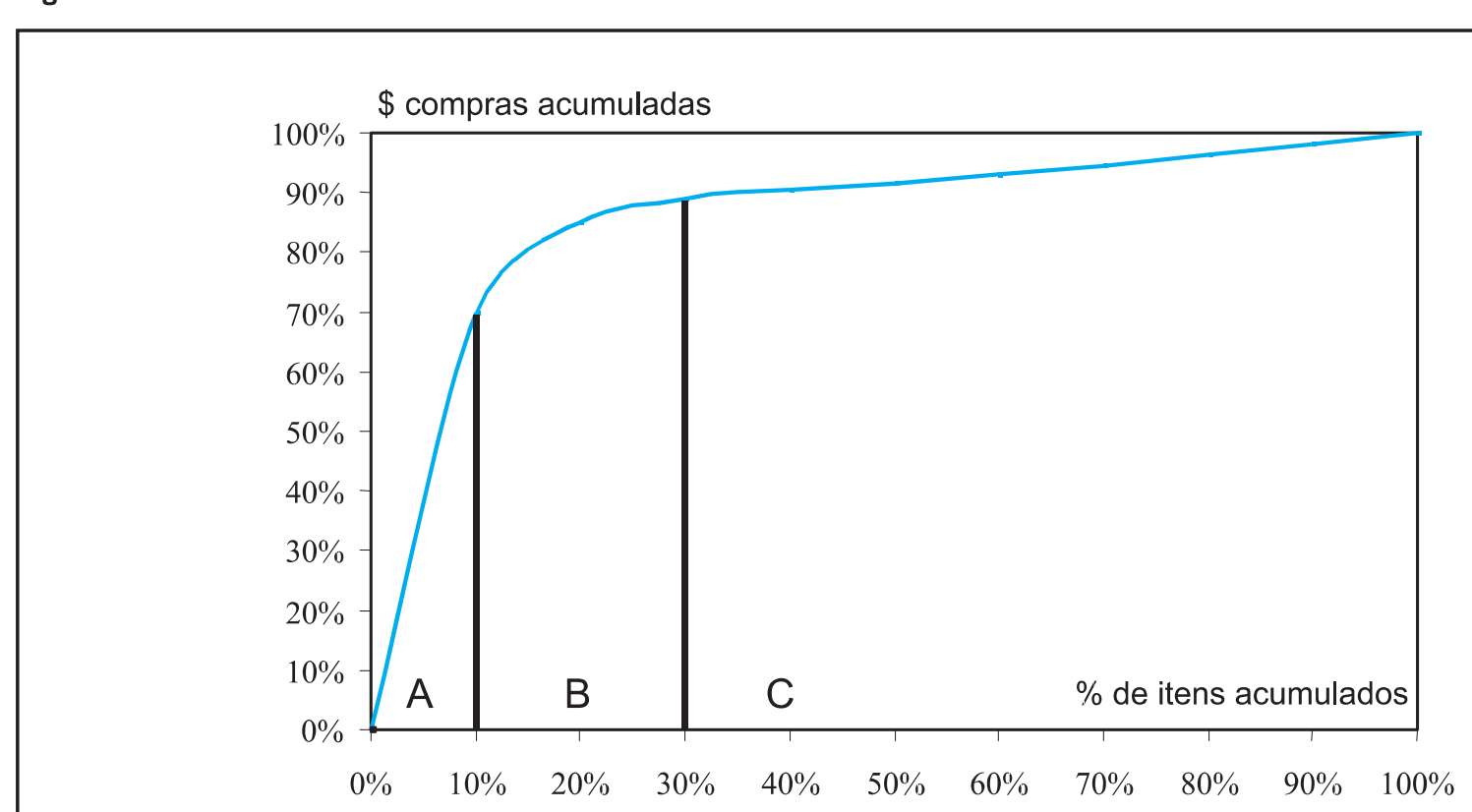


rão de fornecedores, segundo: (i) oportunidade e risco; e (ii) volume de compras. As classes propostas são: (i) altas oportunidade e risco e baixo volume de compras: itens gargalo; (ii) altas oportunidade e risco e alto volume de compras: itens estratégicos; (iii) baixas oportunidade e risco e baixo volume de compras: itens não-críticos; e (iv) baixas oportunidade e risco e alto volume de compras: itens alavancáveis. Itens gargalo são de difícil substituição, operam em mercados monopolizados ou com barreiras de entrada, ou ainda em situação geográfica ou política crítica. Os itens estratégicos são importantes no contexto geral de compras da empresa, de difícil substituição e com poucas alternativas de suprimento. Os itens não-críticos são padronizados, têm suprimento diversificado e são substituíveis. Por fim, os itens alavancáveis têm boa disponibilidade, preços padronizados e são substituíveis. $\mathrm{O}$ autor menciona que maior atenção deva ser dada aos itens estratégicos. É neste quadrante, segundo o autor, que a empresa deve focar o desenvolvimento de fornecedores.

\section{Medição de desempenho em cadeias de suprimentos}

Entende-se que o desempenho de fornecedores possa e deva ser avaliado, não por um ou poucos critérios, mas por uma estrutura multicriterial de mensurações. Algumas incertezas sobre mensuração de desempenho, no entanto, surgem na literatura.

Para Daugherty, Ellinger e Gustin (1996), há incerteza na noção de desempenho, pois algumas vezes não se sabe exatamente o que deve ser medido. Também afirmam que desempenho é uma construção cognitiva complexa e intangível, cuja descrição requer múltiplos indicadores. Pires (2004) define o desempenho como a informação quantifi- cada do resultado de processos, que pode ser comparada com metas, resultados passados e outros processos. Para Neely, Gregory e Platts (1995), indicadores de desempenho individuais devem formar um conjunto maior estruturado em dimensões. Para Sellitto (2005), um sistema de medição de desempenho pode ser pensado como uma estrutura hierarquizada de variáveis de estado, cada uma com a sua metodologia específica de cálculo. Para o autor, tal estrutura permite comparar desempenhos de várias estratégias para os mesmos objetivos de negócios. Para Chow, Heaver e Henriksson (1994), é a informação sobre o desempenho que alinha ações e estratégias: os indicadores são a realimentação entre a competição e a estratégia.

Quanto a cadeias de suprimentos, Simch-Levi, Kaminski e Simch-Levi (2003) acham que estas são redes complexas de instalações e organizações, e que podem abrigar objetivos e estratégias parciais conflitantes. Sempre que partes individuais operam juntas, podem surgir emergências e imprevisibilidades que dificultam a formulação de objetivos comuns e de uma estratégia compartilhada. Como a atual base conceitual da medição de desempenho foi construída para unidades individuais, abre-se uma pauta de pesquisa: lançar novas bases ou modificar as existentes para a medição de desempenho em cadeias de suprimentos.

Segundo Gunasekaran, Patel e Tirtiroglu (2001), os indicadores de desempenho devem considerar os objetivos de toda a cadeia de suprimentos. Para Chow, Heaver e Henriksson (1994), o desempenho da cadeia de suprimentos é multidimensional, envolvendo vários objetivos e exigindo a combinação de diversas medições. Para Pires (2004), antes de especificar as medições, deve-se identificar a estratégia

Figura 2: Representação gráfica da importância estratégica e criticidade.

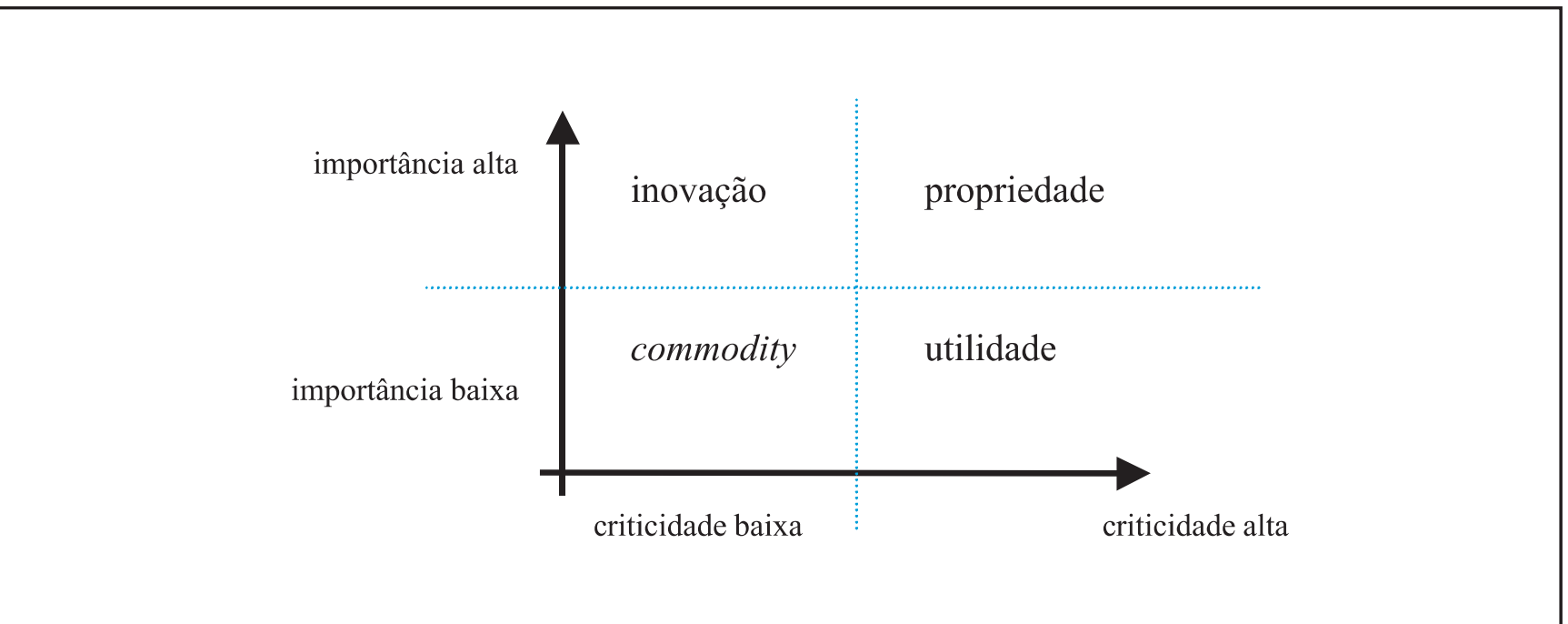

Fonte: Dornier et al., 2000 
da cadeia, o que pode ser difícil, pois uma empresa pode participar de várias cadeias, com estratégias diferentes. Se a competição ocorrer entre cadeias e não entre empresas individuais, a competitividade será construída por uma estratégia que inclui toda a cadeia. Neste caso, as empresas devem manter seus sistemas individuais de medição de desempenho e acrescentar indicadores comuns que reflitam a ação de cadeia. São estes indicadores comuns que alinharão os esforços individuais aos esforços de cadeia e encorajarão as empresas a, além de perseguirem seus objetivos individuais, também perseguirem os objetivos da cadeia.

\section{função compras escolhe e
desenvolve fornecedores que} suportem a estratégia de produção e o
posicionamento competitivo do negócio.

uma parte isolada, seja de um arranjo complexo, deva-se concentrar as medições em um conjunto manejável de indicadores que, eventualmente, produzam um índice final combinado. Tais indicadores devem apreender, com uma dada confiabilidade, uma parte representativa do significado intangível dos construtos latentes visados pela estratégia de operação do objeto em medição.

Um desempenho exige um modelo para mensuração e comunicação, obtido por construção mental. A construção mais abstrata é o termo teórico, que aglutina aspectos de uma definição suficientemente ampla, estruturados em construtos e os conceitos. Os construtos também são construções abstratas, deliberadamente criadas para atender a um propósito científico, porém já mais próximas da realidade. $\mathrm{O}$ conceito, por fim, ainda não é o fenômeno, mas já pode comunicar suas implicações. Suas dimensões são representáveis por valores numéricos, os indicadores, que podem ser combinados e resumidos quantitativamente em índices, segundo esquemas teóricos em hierarquia que auxiliam a representar a realidade

Outras abordagens surgem para lidar com a complexidade da cadeia de suprimentos. Hansen (2004) propõe uma abordagem meso-analítica, que define e descreve um espaço maior do que a empresa e menor do que o ambiente de negócios, no qual ocorrem os fenômenos de cadeias. Gasparetto (2003) propõe uma análise em arborescência, centrada na empresa focal, e ramificada a montante e a jusante. Pires e Aravechia (2001) propõem uma abordagem gráfica em forma de radar, na qual indicadores evoluem, acumulam resultados e são acompanhados ao longo de toda a cadeia. Beamon (1999) prescreve que a avaliação de desempenho de cadeia deva possuir abrangência, universalidade, mensurabilidade, consistência e conectividade. A abrangência mede as pertinências da cadeia; a universalidade compara cadeias; a mensurabilidade garante que os dados sejam facilmente obtidos; a consistência exige que a avaliação reflita a estratégia da cadeia; e a conectividade possibilita que sistemas parciais se conectem, gerando um sistema integrado de avaliação.

Dornier et al. (2000) alertam que a medição do desempenho de cadeias de suprimento deve monitorar, não apenas os processo logísticos, mas também a execução da estratégia e a validade dos pressupostos assumidos na sua formulação. Em suma, os indicadores devem apreender modificações na estratégia e no cenário de competição da cadeia. Pires (2004) cita um trabalho de 1994 em que praticantes, acadêmicos e consultores desenvolveram medidas de desempenho para cadeias, abrangendo: satisfação do cliente, tempo, custo e uso de recursos.

\section{Estruturação de medições}

Entende-se que, para mensurar um desempenho, seja de intangível (SELLITTO e RIBEIRO, 2004; LAKATOS e MARCONI, 1991).

Uma hierarquia é uma construção lógica baseada em entidades agrupadas em níveis. Segundo Saaty (1991), a hierarquia permite explicar os níveis mais altos por ramificações descendentes, em uma lógica de decomposição. Enslinn, Montibeller e Noronha (2001) chamam tais hierarquias de estruturas arborescentes. Sellitto e Walter (2006) chamamnas de estruturas em forma de árvore (tree-like). Um conceito mais complexo é dividido em conceitos menos complexos, arranjados de modo a não haver subordinação múltipla. É necessário determinar a força com que os elementos em um nível influenciam os elementos do nível imediatamente acima, chegando a uma distribuição relativa de impactos. Saaty (1991) sugere brainstorms. Sellitto e Walter (2006) usam grupos focados para listar e ponderar os conceitos influentes no objetivo geral.

Uma estrutura hierárquica é composta por conceitos qualitativos, que podem ser representados numericamente por escalas. Uma escala envolve premissas de relação entre atributos dos entes e a representação simbólica destes atributos por rótulos numéricos. A fidedignidade desta representação é juízo do investigador, apoiado no seu conhecimento da hierarquia e dos entes. Likert propôs uma escala de cinco pontos [ótimo; bom; médio; ruim; péssimo], que tem sido aplicada em mensuração qualitativa. A confiabilidade da mensuração pode ser medida pelo alfa de Crombach, um coeficiente de correlação ao quadrado $\left(\mathrm{R}^{2}\right)$ entre a medida suposta e a medida real do fenômeno estudado, que expressa o quanto a escala descreve o fenômeno (PEREIRA, 1999). 


\section{A PESQUISA: METODOLOGIA E RESULTADOS}

O objetivo principal do artigo é avaliar e classificar, segundo multicritérios de desempenho, dez fornecedores considerados críticos em uma manufatura $O K P$.

$\mathrm{O}$ método de pesquisa é o estudo de caso. O método do caso examina em profundidade um fenômeno contemporâneo, não separando o fenômeno observado do ambiente e levantando hipóteses para futuros estudos. Segundo Eckstein (1975, apud ROESCH, 1999), um estudo de caso pode contribuir para uma teoria de cinco modos. O primeiro modo oferece uma descrição profunda e específica de um objeto. $\mathrm{O}$ segundo modo interpreta eventuais regularidades como evidências de postulados teóricos ainda não verificados. $\mathrm{O}$ terceiro modo é heurístico: uma situação é construída para testar uma idéia. O quarto modo estabelece sondagens plausíveis acerca de uma teoria proposta pelo modo heurístico e o quinto modo é o caso crucial: apóia ou refuta a teoria. Nos três últimos tipos, os objetivos são, em graduação: exploração, geração e teste de teorias. Entende-se que a contribuição do presente estudo de caso é do terceiro tipo, pois foi construída uma situação para o teste de uma idéia.

O método de trabalho foi: (i) Antecedentes: foram identificados os critérios da empresa para avaliação e classificação dos atuais fornecedores; (ii) Definição dos fornecedores estudados: segundo o passo anterior e a base de dados da empresa, foram escolhidos dez fornecedores de componentes para avaliação; (iii) Construção de indicadores de desempenho de fornecedores: pela técnica de grupo focado e análise hierárquica, foram definidos os construtos latentes e os respectivos indicadores de desempenho, chegando-se a uma estrutura multicriterial de desempenho dos fornecedores; (iv) Avaliação: especialistas de compras avaliaram o desempenho dos fornecedores segundo a estrutura definida; (v) Classificação: com os resultados multicriteriais da etapa anterior, separaram-se os fornecedores em aglomerados; e (vi) Discussão: analisando os resultados, têm-se as bases para, na seqüência do trabalho, definir estratégias para os aglomerados.

\section{Resultados \\ 1o Passo: Antecedentes}

A empresa avalia fornecedores segundo critérios de pontualidade de entrega e qualidade, medidos mensalmente e julgados trimestralmente por uma comissão, que define ações corretivas para os fornecedores de mau desempenho. A empresa mantém tratativas individuais com cada fornecedor, constando de análise e ações específicas e exclusivas. Este estudo permitirá a formulação de estratégias padronizadas de gerenciamento de fornecedores, que poderão ser aglomerados por semelhança de desempenho em multicritérios de decisão.

\section{2o Passo: Definição da amostra de fornecedores críticos}

A empresa opera com mais de seiscentos fornecedores cadastrados e classificados, dos quais escolheram-se dez para compor a amostra que será estudada. Os fornecedores escolhidos são os de maior complexidade e criticidade no suprimento, isto é, itens de escassas alternativas de aquisição, alta importância no produto e alto valor econômico. Em suma, fornecedores classe A que fornecem itens de propriedade, como no nível IV da Figura 3.

Figura 3: Matriz de criticidade de materiais.

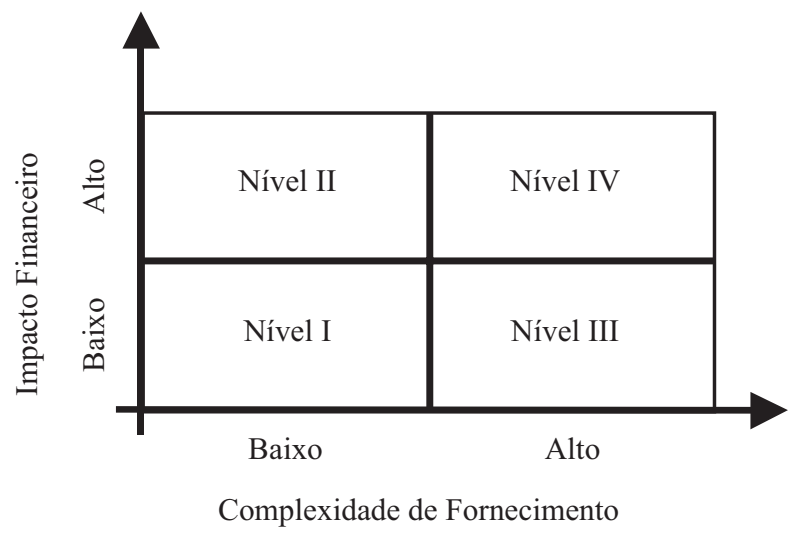

Fonte: sistema de informação da empresa. 
Os fornecedores escolhidos e seus itens são: (i) WSI: papel Pressphan; (ii) WB: componentes isolantes; (iii) ABP: buchas capacitivas de alta tensão; (iv) IEMM: ventiladores, radiadores e válvulas de refrigeração; (v) PC: fios de cobre isolado e cabos transpostos; (vi) CI: tanques, tampas e armaduras; (vii) TK: aço silício; (viii) PV: buchas capacitivas de alta tensão; (ix) MRB: comutadores sob e sem carga; e (x) ABL: buchas capacitivas de alta tensão.

3o passo: Construção dos indicadores de desempenho de fornecedores

Um grupo focado de seis estrategistas de suprimentos da empresa desmembrou em construtos o termo teórico - desempenho em suprimento. Usou-se a técnica apresentada em Ribeiro e Newman (2003). As perguntas foram: (i) Como se dá a relação entre consumidor e fornecedor? (ii) No âmbito empresarial, como definir esta relação? (iii) Quais os atributos, na sua opinião, que uma empresa deve ter para ser considerada um excelente fornecedor? (iv) $\mathrm{Na}$ sua opinião, para que tipo de relação deverá evoluir a convivência hoje existente entre fornecedor e comprador em uma empresa de fabricação sob encomenda?

Anotações foram feitas, identificando ênfases, necessidades e pontos em comum. Identificaram-se construtos apontados pelos participantes sob formas semânticas diversas: (i) qualidade; (ii) preço; (iii) entrega; (iv) flexibilidade; e (v) tecnologia. Tais construtos diferem pouco das dimensões de competição anteriormente revisadas.

A distribuição de importâncias relativas dos construtos perante o termo teórico desempenho em suprimento foi obtida pelo AHP, aplicado individualmente aos estrategistas. Foi usada uma planilha eletrônica, que fornece o vetor de ponderações e a razão de consistência $C R$, a probabilidade de que a distribuição de importâncias seja aleatória, não se originando, portanto, de um julgamento. Julgamentos foram refeitos sempre que $C R>0,1$. Ao fim, calcularamse as médias das importâncias dos construtos e seus coeficientes de variação, informados na Tabela 1 . O grupo associou dois indicadores com pesos iguais aos construtos qualidade, preço, entrega, tecnologia e flexibilidade, como no Quadro 1.

\section{$4^{\circ}$ passo: Avaliação dos fornecedores}

A avaliação dos fornecedores foi realizada por questionário, respondido por cinco dos seis participantes do grupo focado, os compradores. A cada quesito, os avaliadores opinaram em que nível o fornecedor se encontra [ $1=$ decepciona totalmente; 2 = decepciona parcialmente; $3=$ médio; $4=$ atende parcialmente; $5=$ atende plenamente]. Alguns respondentes se omitiram em avaliar alguns dos fornecedores por desconhecer sua situação ou por nunca terem negociado com eles. As respostas são apresentadas nas Tabelas 2 e 3 .

A confiabilidade dos resultados foi verificada pelo alfa de Crombach, calculado pelo software SPSS for Windows, v 8.0.0, 1997. Como o teste exige respostas completas e houve omissões, ocuparam-se as lacunas com as médias dos outros respondentes. Foram feitos cinco testes, um por construto, constando cada teste de vinte variáveis e cinco casos. Os casos correspondem aos cinco respondentes e as variáveis às vinte avaliações por construto (dois indicadores por construto para dez fornecedores). Detalhes do teste surgem em Hair et al. (1998).

Os alfas dos construtos são: (i) qualidade $=0,56$; (ii) preço $=0,57$; (iii) entregas $=0,71$; (iv) tecnologia $=0,74$;

Quadro 1: Indicadores por construto.

\begin{tabular}{|l|l|}
\hline \multicolumn{1}{|c|}{ CONSTRUTO } & \multicolumn{1}{|c|}{ INDICADOR } \\
\hline Qualidade & $\begin{array}{l}\text { Integridade da entrega } \\
\text { Atendimento } \\
\text { Preço }\end{array}$ \\
& $\begin{array}{l}\text { Relação com o mercado } \\
\text { Prazo de pagamento }\end{array}$ \\
Entrega & $\begin{array}{l}\text { Pontualidade } \\
\text { Prazo }\end{array}$ \\
Tecnologia & Processo \\
& Produto \\
Flexibilidade & Volume \\
& Produto \\
\hline
\end{tabular}

Tabela 1: Resultado da aplicação do AHP.

\begin{tabular}{|c|c|c|c|c|c|c|c|c|}
\hline CONSTRUTOS & AVAL. 1 & AVAL. 2 & AVAL. 3 & AVAL. 4 & AVAL, 5 & AVAL. 6 & MÉDIA & $\mathbf{C V}=\sigma / \mu$ \\
\hline Qualidade & $12,9 \%$ & $24,3 \%$ & $42,0 \%$ & $15,7 \%$ & $47,8 \%$ & $41,8 \%$ & $30,8 \%$ & 0,49 \\
\hline Entrega & $26,1 \%$ & $26,7 \%$ & $16,2 \%$ & $26,9 \%$ & 23,3\% & $26,7 \%$ & $24,3 \%$ & 0,17 \\
\hline Flexibilidade & $3,3 \%$ & $11,3 \%$ & $5,8 \%$ & $10,0 \%$ & $7,3 \%$ & $4,6 \%$ & $7,1 \%$ & 0,44 \\
\hline Preço & $51,3 \%$ & $26,5 \%$ & $25,3 \%$ & $42,2 \%$ & $17,5 \%$ & $9,7 \%$ & $28,7 \%$ & 0,54 \\
\hline Tecnologia & $6,3 \%$ & $11,2 \%$ & $10,7 \%$ & $5,1 \%$ & $4,1 \%$ & $17,1 \%$ & $9,1 \%$ & 0,54 \\
\hline Total & $100 \%$ & $100 \%$ & $100 \%$ & $100 \%$ & $100 \%$ & $100 \%$ & $100 \%$ & \\
\hline
\end{tabular}


Tabela 2: Respostas dos avaliadores, primeira parte.

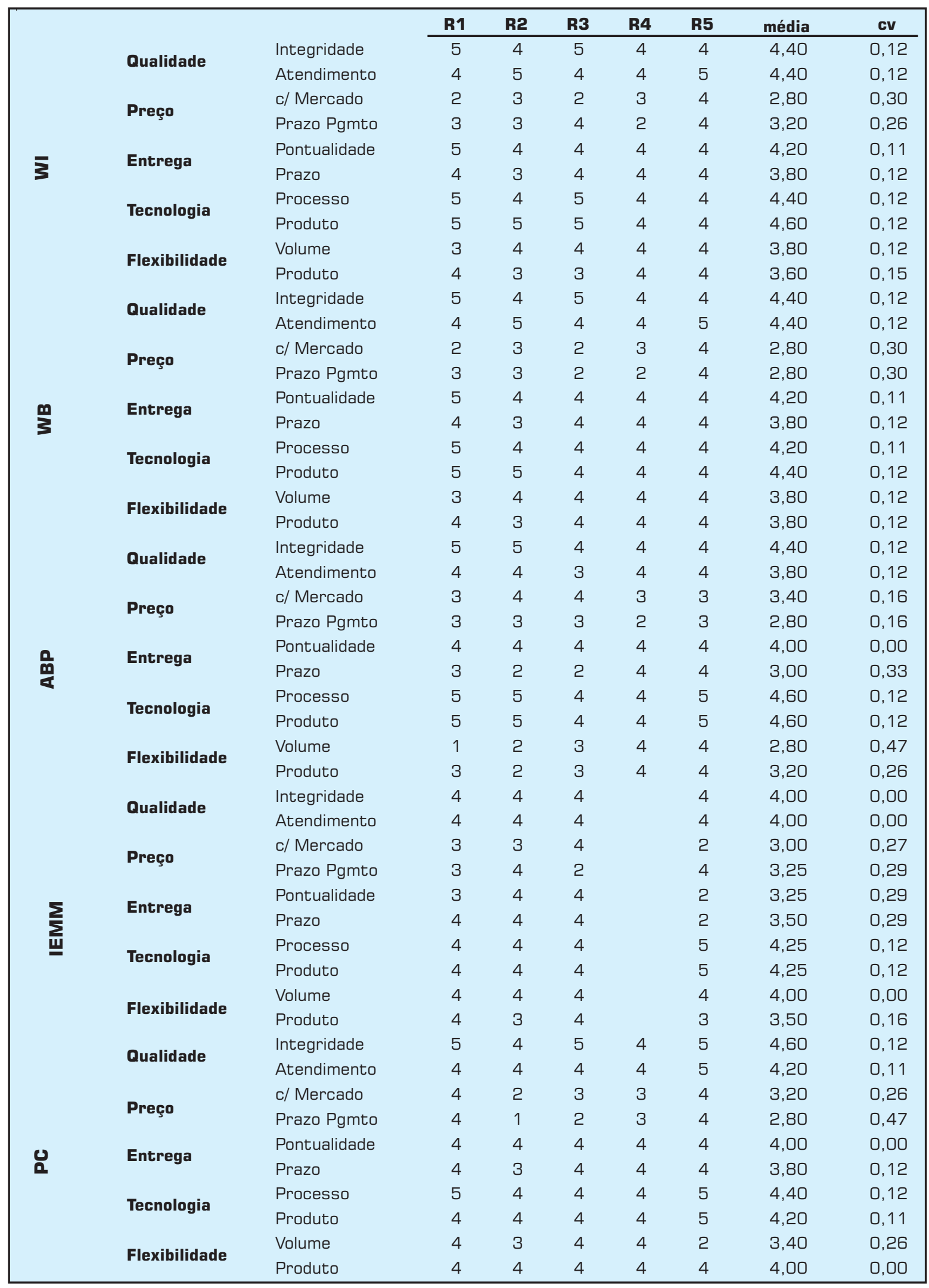


Tabela 3: Respostas dos avaliadores, segunda parte.

\begin{tabular}{|c|c|c|c|c|c|c|c|c|c|}
\hline \multirow{13}{*}{$\overline{0}$} & & & R1 & $\mathbf{R 2}$ & R3 & R4 & R5 & média & cv \\
\hline & \multirow{2}{*}{ Qualidade } & Integridade & 3 & 2 & 2 & & 4 & 2,75 & 0,35 \\
\hline & & Atendimento & 2 & 3 & 2 & & 2 & 2,25 & 0,22 \\
\hline & \multirow{2}{*}{ Preço } & c/ Mercado & 4 & 5 & 4 & & 5 & 4,50 & 0,13 \\
\hline & & Prazo Pgmto & 4 & 4 & 2 & & 4 & 3,50 & 0,29 \\
\hline & \multirow{2}{*}{ Entrega } & Pontualidade & 3 & 3 & 2 & & 2 & 2,50 & 0,23 \\
\hline & & Prazo & 4 & 4 & 4 & & 4 & 4,00 & 0,00 \\
\hline & \multirow{2}{*}{ Tecnologia } & Processo & 3 & 2 & 3 & & 4 & 3,00 & 0,27 \\
\hline & & Produto & 3 & 3 & 3 & & 4 & 3,25 & 0,15 \\
\hline & \multirow{2}{*}{ Flexibilidade } & Volume & 4 & 4 & 4 & & 4 & 4,00 & 0,00 \\
\hline & & Produto & 3 & 4 & 4 & & 4 & 3,75 & 0,13 \\
\hline & \multirow{2}{*}{ Qualidade } & Integridade & & 5 & 4 & 4 & 4 & 4,25 & 0,12 \\
\hline & & Atendimento & & 4 & 3 & 4 & 4 & 3,75 & 0,13 \\
\hline \multirow{8}{*}{$\underline{Y}$} & \multirow{2}{*}{ Preço } & c/ Mercado & & 4 & 2 & 3 & 4 & 3,25 & 0,29 \\
\hline & & Prazo Pgmto & & 5 & 4 & 4 & 4 & 4,25 & 0,12 \\
\hline & \multirow{2}{*}{ Entrega } & Pontualidade & & 4 & 4 & 4 & 4 & 4,00 & 0,00 \\
\hline & & Prazo & & 2 & 2 & 4 & 4 & 3,00 & 0,38 \\
\hline & \multirow{2}{*}{ Tecnologia } & Processo & & 4 & 4 & 4 & 4 & 4,00 & 0,00 \\
\hline & & Produto & & 4 & 4 & 4 & 5 & 4,25 & 0,12 \\
\hline & \multirow{2}{*}{ Flexibilidade } & Volume & & 3 & 4 & 4 & 2 & 3,25 & 0,29 \\
\hline & & Produto & & 3 & 2 & 4 & 4 & 3,25 & 0,29 \\
\hline \multirow{11}{*}{ 文 } & \multirow{2}{*}{ Qualidade } & Integridade & 4 & 4 & 4 & 4 & 4 & 4,00 & 0,00 \\
\hline & & Atendimento & 4 & 4 & 5 & 4 & 3 & 4,00 & 0,18 \\
\hline & \multirow{2}{*}{ Preço } & c/ Mercado & 4 & 3 & 3 & 3 & 3 & 3,20 & 0,14 \\
\hline & & Prazo Pgmto & 4 & 4 & 5 & 4 & 3 & 4,00 & 0,18 \\
\hline & \multirow{2}{*}{ Entrega } & Pontualidade & 3 & 4 & 4 & 4 & 2 & 3,40 & 0,26 \\
\hline & & Prazo & 3 & 2 & 2 & 4 & 2 & 2,60 & 0,34 \\
\hline & \multirow{2}{*}{ Tecnologia } & Processo & 4 & 4 & 4 & 4 & 4 & 4,00 & 0,00 \\
\hline & & Produto & 3 & 4 & 4 & 4 & 4 & 3,80 & 0,12 \\
\hline & \multirow{2}{*}{ Flexibilidade } & Volume & 3 & 4 & 4 & 4 & 2 & 3,40 & 0,26 \\
\hline & & Produto & 3 & 3 & 4 & 4 & 2 & 3,२० & 0,26 \\
\hline & \multirow{2}{*}{ Qualidade } & Integridade & 5 & 4 & 5 & 4 & 4 & 4,40 & 0,12 \\
\hline & & Atendimento & 5 & 4 & 2 & 4 & 4 & 3,80 & 0,29 \\
\hline & Prean & c/ Mercado & 2 & 3 & 2 & 2 & 3 & 2,40 & 0,23 \\
\hline & Feçర & Prazo Pgmto & 2 & 3 & 4 & 2 & 3 & 2,80 & 0,30 \\
\hline & Entrega & Pontualidade & 5 & 4 & 4 & 4 & 4 & 4,20 & 0,11 \\
\hline$\underline{\alpha}$ & Entrega & Prazo & 4 & 3 & 2 & 4 & 4 & 3,40 & 0,26 \\
\hline & Tecnologia & Processo & 5 & 4 & 4 & 4 & 4 & 4,20 & 0,11 \\
\hline & Tecnologla & Produto & 4 & 4 & 5 & 4 & 4 & 4,20 & 0,11 \\
\hline & Flexibilidade & Volume & 3 & 3 & 2 & 4 & 4 & 3,२० & 0,26 \\
\hline & FIexidiliadue & Produto & 3 & 3 & 2 & 4 & 4 & 3,20 & 0,26 \\
\hline & Oualidade & Integridade & 5 & 4 & 4 & 4 & 4 & 4,20 & 0,11 \\
\hline & Gualidade & Atendimento & 4 & 4 & 3 & 4 & 4 & 3,80 & 0,12 \\
\hline & Preco & c/ Mercado & 4 & 4 & 4 & 3 & 3 & 3,60 & 0,15 \\
\hline & Preço & Prazo Pgmto & 4 & 3 & 2 & 3 & 3 & 3,00 & 0,24 \\
\hline & Entreaa & Pontualidade & 4 & 4 & 3 & 4 & 2 & 3,40 & 0,26 \\
\hline$\vec{m}$ & & Prazo & 3 & 2 & 2 & 4 & 4 & 3,00 & 0,33 \\
\hline & Tecnoloaia & Processo & 5 & 4 & 4 & 4 & 4 & 4,20 & 0,11 \\
\hline & & Produto & 5 & 5 & 4 & 4 & 4 & 4,40 & 0,12 \\
\hline & Flexibilidade & Volume & 4 & 3 & 3 & 4 & 4 & 3,60 & 0,15 \\
\hline & 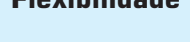 & Produto & 3 & 3 & 3 & 4 & 2 & 3,00 & 0,24 \\
\hline
\end{tabular}


e (v) flexibilidade $=0,77$. Segundo Hair et al. (1998), em pesquisas exploratórias, o alfa deve ser maior do que 0,50. Malhotra (1999) fala em 0,60. Os construtos qualidade e preço estão mais próximos destes limites e parecem menos compreendidos pelos respondentes. Em outras aplicações, o que se espera medir nestes construtos deverá ser mais bem discutido nas sessões de grupo focado para que se obtenham confiabilidades mais altas.

5o passo: Classificação dos fornecedores por análise de aglomerados

Os resultados da avaliação, ponderados pelas importâncias relativas calculadas pelo AHP (Tabela 1), são apresentados na Tabela 4. O critério Qualidade, por exemplo, recebeu uma importância relativa de 30,8\%. O fornecedor WI recebeu avaliações de 4,4 e 4,4 nos dois indicadores de qualidade. A soma de produtos $[0,308.4,4+0,308.4,4]=2,71$ é o escore do fornecedor WI para o critério. A soma dos escores parciais compõe o escore total, usado para formar a ordem, apontada na última coluna da tabela. O maior escore possível é 10.

Estes dados foram utilizados para, com a ajuda do software SPSS for Windows, v 8.0.0, 1997, pelo método k-means, obter as aglomerações por semelhança de desempenhos da Tabela 5.

Tabela 4: Escores parciais e totais dos fornecedores.

\begin{tabular}{|c|c|c|c|c|c|c|c|}
\hline \multirow{2}{*}{ FORNECEDOR } & \multicolumn{6}{|c|}{ ESCORES } & \multirow{2}{*}{ ORDEM } \\
\hline & QUALIDADE & PREÇO & ENTREGA & TECNOLOGIA & FLEXIBILIDADE & TOTAL & \\
\hline W1 & 2,71 & 1,46 & 0,56 & 2,59 & 0,67 & 7,99 & 1 \\
\hline WB & 2,71 & 1,36 & 0,56 & 2,47 & 0,69 & 7,80 & 3 \\
\hline ABP & 2,52 & 1,51 & 0,49 & 2,65 & 0,55 & 7,71 & 5 \\
\hline IEMM & 2,46 & 1,52 & 0,48 & 2,44 & 0,68 & 7,58 & 7 \\
\hline PC & 2,71 & 1,46 & 0,55 & 2,47 & 0,67 & 7,86 & 2 \\
\hline $\mathrm{Cl}$ & 1,54 & 1,95 & 0,46 & 1,80 & 0,70 & 6,44 & 10 \\
\hline TK & 2,46 & 1,82 & 0,49 & 2,37 & 0,59 & 7,74 & 4 \\
\hline PV & 2,46 & 1,75 & 0,42 & 2,24 & 0,60 & 7,48 & 8 \\
\hline MRB & 2,52 & 1,26 & 0,54 & 2,42 & 0,58 & 7,32 & 9 \\
\hline$A B L$ & 2,46 & 1,60 & 0,45 & 2,47 & 0,60 & 7,59 & 6 \\
\hline
\end{tabular}

Tabela 5: Formação dos aglomerados.

\begin{tabular}{|c|c|c|c|c|c|c|c|}
\hline \multirow{2}{*}{\multicolumn{2}{|c|}{ FORNECEDOR }} & \multicolumn{6}{|c|}{ PERTINÊNCIAS A AGLOMERADOS } \\
\hline & & \multicolumn{2}{|c|}{ DOIS AGLOMERADOS } & \multicolumn{2}{|c|}{ TRÊS AGLOMERADOS } & \multicolumn{2}{|c|}{ QUATRO AGLOMERADOS } \\
\hline NOME & CASO & AGLOMERADO & $\begin{array}{l}\text { DISTÂNOIA AO } \\
\text { GENTRO }\end{array}$ & AGLOMERADO & $\begin{array}{l}\text { DISTÂNCIA AO } \\
\text { CENTRO }\end{array}$ & AGLOMERADO & $\begin{array}{l}\text { DISTÂNCIA AO } \\
\text { CENTRO }\end{array}$ \\
\hline W1 & 1 & 2 & 0,300 & 2 & 0,300 & 1 & 0,000 \\
\hline WB & 2 & 2 & 0,000 & 2 & 0,000 & 1 & 0,300 \\
\hline ABP & 3 & 2 & 1,077 & 3 & 1,044 & 3 & 0,000 \\
\hline IEMM & 4 & 2 & 0,811 & 2 & 0,811 & 1 & 0,791 \\
\hline PC & 5 & 2 & 0,245 & 2 & 0,245 & 1 & 0,224 \\
\hline $\mathrm{Cl}$ & 6 & 1 & 0,000 & 1 & 0,000 & 2 & 0,000 \\
\hline TK & 7 & 2 & 1,282 & 3 & 0,573 & 4 & 0,573 \\
\hline PV & 8 & 2 & 1,487 & 3 & 0,000 & 4 & 0,000 \\
\hline MRB & 9 & 2 & 0,735 & 2 & 0,735 & 3 & 0,735 \\
\hline $\mathrm{ABL}$ & 10 & 2 & 1,140 & 3 & 0,539 & 4 & 0,539 \\
\hline
\end{tabular}




\section{DISCUSSÃO}

Discutem-se, a seguir, aspectos relevantes ligados ao método e ao resultado do caso. uma técnica mais identificada com a chamada linha fenomenológica de pesquisa, o grupo focado, com técnicas mais identificadas com a linha positivista, o questionário fechado. $\mathrm{O}$ uso das entrevistas qualitativas em grupo focado explorou as percepções individuais e únicas de estrategistas acerca das exigências de desempenho em suprimentos. Ao fim, chegouse a uma estrutura de desempenho que pode ser mensurada por instrumento padronizado, o questionário.

Observou-se, durante a aplicação da técnica, a ação de um grupo maduro, que se manifesta espontânea e sinceramente, defende opiniões e identifica serenamente suas divergências, o que facilitou o trabalho. Entende-se que a seleção do grupo, composto por estrategistas de sucesso na empresa e especialistas na atividade, contribuiu para o sucesso da tarefa.
Quanto ao método, observa-se que houve a integração de

dadas duas alternativas, o decisor escolhe a mais atrativa e classifica as diferenças de atratividade em relação a critérios (fraca, moderada, forte, etc..). Por modelos de programação linear, o método calcula ponderações e sugere revisão de julgamentos. Segundo Mello, Gomes e Lins (2002), o método pode produzir um julgamento consistente. Em sua revisão, estes autores citam Bana e Costa e Vasnick (1994; 1995). Já os proponentes do AHP admitem $10 \%$ de inconsistência, mas este valor pode ser reduzido medindo a $C R$ e revisando os julgamentos que produzem a inconsistência, como foi feito no presente caso. Por ora interessa como contribuição essencial a formulação do problema, destacando-se que os resultados do caso podem ser modificados caso se use outro método. Não é objetivo do artigo ir além das considerações que já fazem Machado, Gomes e Chauvel (2003).

Adotaram-se as médias das preferências dos estrategistas como resultado final. Observa-se, no entanto, que os coeficientes de variação são relevantes e representam as diferenças de opinião entre estrategistas, o que enfatiza a subjetividade presente na avaliação e reforça o uso de técnicas da linha fenomenológica em engenharia de produção. Três fornecedores não foram avaliados por alguns entrevistados, que alegaram não ter base para uma avaliação consistente para a avaliação. Tal fato sugere que, em outras aplicações, este aspecto seja tratado ante-

Ainda no âmbito do grupo focado, observou-se que a utilização do AHP auxiliou na quantificação das importâncias relativas dos construtos no termo teórico, possibilitando a mensuração de subjetividades. O modo hierárquico de pensar ajudou o grupo a transformar idéias e impressões pessoais em uma estrutura mensurável por meios padronizados. Quando houve inconsistências em julgamentos, adotou-se o critério arbitrário de Saaty (1991): revisão de julgamentos com $C R$ maior que 0,1 . Com isto, não se admitem inconsistências maiores do que $10 \%$ nos julgamentos que fundamentam a estrutura de avaliação de desempenho.

O AHP, apesar de abundantemente exemplificado na literatura (PARTOVI, WHITERS e BRADFORD, 2002), sofre críticas. Dyer (1990a; 1990b) aponta alterações na ordenação quando alternativas são adicionadas. Saaty (1990) e Harker e Vargas (1990) replicam. Sellitto (2005) aponta e discute o mesmo fenômeno quando um critério é retirado. Machado, Gomes e Chauvel (2003) resenham e comentam as críticas apresentadas ao método. Gomes, Araya e Carignano (2004) e Liu e Hai (2005) apresentam alternativas que modificam o método. Mello, Gomes e Lins (2002) comparam o AHP ao método MACBETH (Measuring Attractiveness by a Categorigal Based Evaluation Technique). Este, tal como o AHP, fornece uma medida de desempenho de alternativas perante multicritérios de decisão e vale-se de comparações pareadas: cipadamente, pois houve a necessidade de procedimentos compensatórios. Neste trabalho optou-se por usar dois indicadores de mesmo peso para cada construto. Em trabalhos futuros, pode-se aumentar este número e estender a distribuição de importância relativa para dentro dos construtos, chegando-se a uma árvore com entes e influências mais detalhadas, pois as ponderações incluiriam todos os níveis.

O uso de questionários baseados na escala de Likert e de métodos multivariados são recorrentes na literatura e não se justificam ulteriores comentários.

Passa-se agora a comentar aspectos ligados ao resultado do caso.

Os construtos identificados, qualidade, flexibilidade, preço, entrega e tecnologia, apresentam uma relação com o exposto na fundamentação teórica, em que autores citam, de diferentes maneiras, estes critérios de competitividade em operações. Por exemplo, o construto tecnologia deste estudo pode ser comparado aos construtos serviços e inovatividade da revisão bibliográfica, já que estes apreendem principalmente a existência e a efetividade de recursos tecnológicos na estratégia de operação, como o faz aquele nas operações de produção das empresas fornecedoras avaliadas. Essa relação reforça a impressão de efetividade da técnica ora utilizada.

A identificação dos fornecedores foi baseada nos critérios já usados pela empresa, o que produziu resultados satisfa- 
tórios, segundo os especialistas. O escore de desempenho destes fornecedores poderia ter sido usado em uma etapa anterior, que a empresa já percorreu: a escolha dos membros e a formação da cadeia de suprimentos. Observa-se, por exemplo, que ABP, PV e ABL, cujos escores são 7,71; 7,48 e 7,59 , oferecem o mesmo produto, buchas capacitivas de alta tensão, e são concorrentes. Caso fosse necessária uma escolha e a ABP tivesse capacidade para produzir integralmente o suprimento requerido pela manufatura, teria a preferência para entrar na cadeia de suprimentos.

Quanto à aglomeração, esta permite especificar poucas políticas ou estratégias de compras, uma por aglomerado. Watts, Kim e Hahn (1992) definem política ou estratégia de compras como um conjunto de decisões e escolhas relacionadas à aquisição dos materiais e serviços requeridos pela manufatura. Os autores acrescentam que, quando existe a externalização, as prioridades de competição presentes na estratégia de manufatura devem ser sustentadas pela estratégia de compras. A formulação dos planos detalhados integrantes das estratégias dos aglomerados é remetida à continuidade. Por ora, faz-se uma exploração qualitativa inicial nas separações obtidas e uma análise gráfica sobre um dos

Quadro 2: Formação de dois aglomerados.

\begin{tabular}{|c|c|c|}
\hline AGLOMERADO 1 & \multicolumn{2}{|c|}{ AGLOMERADO 2} \\
\hline CI & WI & TK \\
& WB & PV \\
& ABP & MRB \\
& IEMM & ABL \\
& PC & \\
\hline
\end{tabular}

aglomerados resultantes da separação. Na exploração, serão apontados objetivos iniciais para algumas das prioridades padronizadas, segundo o desempenho médio do aglomerado. As aglomerações surgem nos Quadros 2, 3 e 4.

A separação em dois aglomerados isolou CI dos demais fornecedores, devido ao seu mau desempenho no critério qualidade. CI fornece itens de construção mecânica e tem apresentado problemas de qualidade no processo de fabricação. Como CI mantém um bom relacionamento comercial, tem praticado preços atraentes e demonstrado receptividade a críticas, um objetivo estratégico para CI é gerenciar seu desenvolvimento e corrigir seu desempenho em qualidade.

Na separação em três aglomerados, além de CI, surgem dois aglomerados. O aglomerado 2 apresenta: alta ou boa qualidade; maus preços; boa e ruim entrega; médias tecnologia e flexibilidade. À exceção da entrega, os demais critérios apresentam alguma uniformidade. Explica-se a qualidade favorável e os maus preços pela alta especialização e a baixa concorrência presente no aglomerado, principalmente nos materiais de alta tensão de WI, WB, MRB e ABL. Uma estratégia de aglomerado teria como objetivo reduzir custos de produção e melhorar preços, sem afetar os demais construtos. No aglomerado 3, TK e PV têm iguais desempenhos em todos os critérios (bom em preço, médio nos demais) e ABP tem desempenho médio em preço e bom em tecnologia. TK e PV são detentoras de grande mercado e alta escala de produção, portanto, podem ter bom desempenho em preços. ABP é uma multinacional com alta tecnologia, mas com alto custo de produção. Uma estratégia para o aglomerado teria como objetivo inicial melhorar qualidade, entrega, tecnologia e flexibilidade, porém sem elevar custos, para poder manter os preços atuais.

\section{Quadro 3: Formação de três aglomerados.}

\begin{tabular}{|c|c|c|}
\hline AGLOMERADO 1 & AGLOMERADO 2 & AGLOMERADO 3 \\
\hline CI & WI & TK \\
& WB & PV \\
& IEMM & \\
& PC & \\
\hline
\end{tabular}

Quadro 4: Formação de quatro aglomerados.

\begin{tabular}{|c|c|c|c|}
\hline AGLOMERADO 1 & AGLOMERADO 2 & AGLOMERADO 3 & AGLOMERADO 4 \\
\hline WI & CI & ABP & TK \\
WB & & MRB & ABL \\
IEMM & & & \\
\hline
\end{tabular}


Na separação em quatro aglomerados, o aglomerado 1 tem: qualidade ótima; preços ruins; entregas boas; tecnologia boa e flexibilidade ótima. Uma estratégia para o aglomerado teria objetivos de redução de custos e conseqüentemente de preços. No aglomerado 3, ABP e MRB têm qualidade e flexibilidade médias, diferindo nos demais construtos. No aglomerado 4, ABL tem desempenho parecido com ABP na separação anterior, portanto este aglomerado não difere do aglomerado 3, criado na separação em três aglomerados.

Para encerrar a discussão, escolheu-se, para uma análise gráfica, um dos aglomerados, o aglomerado 1 (W1, WB, ABP e PC) do Quadro 4. Escolheu-se este aglomerado, pois seus membros, entre os dez fornecedores avaliados, apresentam quatro dos cinco melhores escores totais. A Figura 4 relaciona o desempenho médio dos membros do aglomerado nos critérios de desempenho e a importância relativa destes critérios.

Os critérios qualidade e tecnologia ocupam a área de excelência, enquanto que, nos demais critérios, não há nem excesso nem escassez na distribuição de recursos. Fica a impressão de que o uso de recursos estratégicos no aglomerado está equilibrado, pois os critérios de alta importância, qualidade e tecnologia, têm desempenho superior; não há critério de alta importância com baixo desempenho (zona de carência) nem critério de baixa importância com alto desempenho (zona de excesso). A distribuição de pontos ao longo da diagonal aponta para o equilíbrio na estratégia e indica que, em média, as estratégias dos membros do aglomerado estão alinhadas com os objetivos estratégicos da empresa focal. Um padrão estratégico observado no aglomerado é: excelência em qualidade e tecnologia e normalidade em preço, flexibilidade e entrega. Esta análise é similar à apresentada em Slack (2002).

\section{CONCLUSÃO}

Este trabalho aplicou, em engenharia de produção, uma técnica mais identificada com a linha fenomenológica de pesquisa, o grupo focado, adequado para análises exclusivas de objetos. O grupo focado fez parte de uma metodologia de trabalho que também contou com técnicas mais identificadas com a linha de pesquisa positivista, adequadas para análises padronizadas de populações de objetos. A técnica do grupo focado utilizou a diversidade de opiniões e impressões de especialistas em aquisições de uma manufatura que externaliza partes, na obtenção de uma estrutura padronizada para a medição de um objeto intangível e multidimensional, o desempenho de fornecedores de itens para a manufatura.

O objetivo de avaliar o desempenho e classificar em aglomerados os fornecedores de uma manufatura $O K P$ foi alcançado. Tal objetivo é representativo, pois tanto a estratégia de aquisições da empresa compradora como a estratégia de produção das empresas fornecedoras, são objetos intangíveis e o alinhamento entre ambas requer a identificação de fatores padronizados de mensuração, comparação e eventuais reformulações em atividades.

A metodologia proposta chegou a resultados que permitem que a empresa modifique o gerenciamento de fornecedores críticos, abrindo a possibilidade de não mais os tratar como indivíduos isolados e com peculiaridades próprias, mas como populações de empresas com características semelhantes, tratáveis por poucas políticas padronizadas. En-

Figura 4: Análise gráfica estratégica de aglomerado.

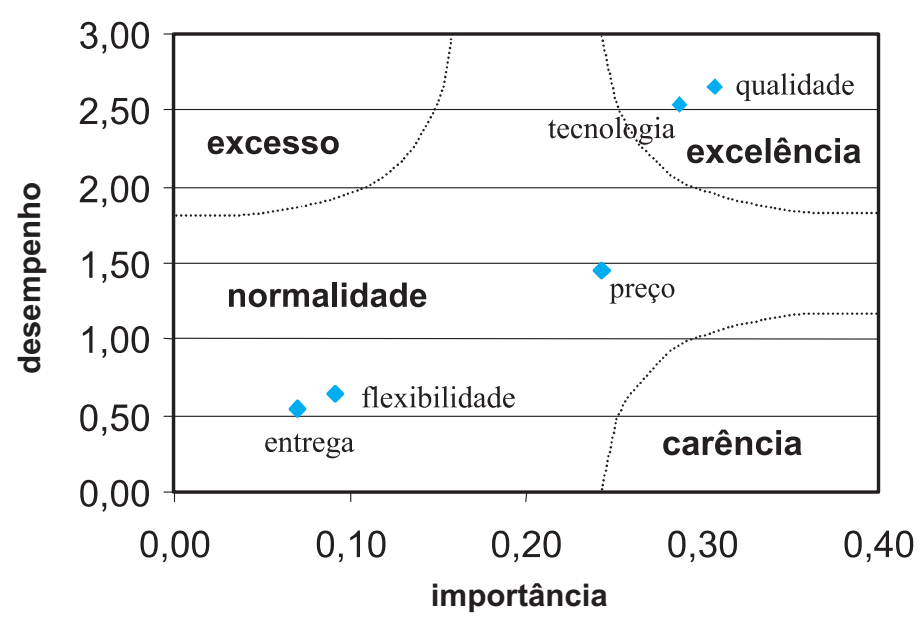


tende-se que esta tenha sido a principal contribuição deste artigo. Também entende-se que a principal opção de continuidade seja a formulação de uma população de estratégias para a gestão da cadeia de suprimentos. Outras opções de continuidade foram apontadas na discussão.

O procedimento proposto sugere a identificação de aglo- merados e a formalização de populações de estratégias, alocáveis a aglomerados possíveis de serem identificados. Beinhocker (1999) e Lowson (2002) apresentam o conceito de população de estratégias, que já foram explorados inicialmente em Sellitto (2005) e que podem ser mais explorados em conjunto com a presente metodologia.

\section{Artigo recebido em 21/12/2005 Aprovado para publicação em 20/10/2006}

\section{- Reconhecimento}

Deseja-se reconhecer a contribuição dada pelos referees anônimos à forma final do artigo.

\section{- Referências Bibliográficas}

ANDRADE, C.; FURTADO, J. Discutindo processos de outsourcing da manufatura: uma análise a partir de elementos das indústrias eletrônica, farmacêutica e automobilística. Anais do XXV ENEGEP, P. Alegre, 2005

ARNOLD, J. Administração de materiais uma introdução. S. Paulo: Atlas,1999.

BROWN, R. Decision rules for inventory management. EUA: Arthur D. Little, 1967.

BEINHOCKER. E. Robust adaptative strategies. Sloan Management Review, v. 40, n. 3, p. 95-106, 1999.

CHEN, I.; PAULRAJ, A.; LADO, A. Strategic purchasing, supply management, and firm performance. Journal of Operations Management, n. 22, p. 505-523, 2004.

CHOW, G.; HEAVER, T.; HENRIKSSON, L. Logistics Performance: Definition and Measurement. International Journal of Physical Distribution \& Logistics Management, v. 24, n. 1, p. 17-28, 1994

CONTADOR, J. Modelo para aumentar a competitividade industrial. S. Paulo: Edgard Blücher, 1996

CRISTOPHER, M. Logística e gerenciamento da cadeia de suprimentos. S. Paulo: Pioneira Thomson Learning, 2002.
DAUGHERTY, P.; ELLINGER, A.; GUSTIN, G. Integrated logistics: achieving logistics performance improvements. Supply Chain Management, v. 1, n. 3, p. 25-33, 1996

DAVIS, M : AQUILANO, N : CHASE, R. Fundamentos da Administração da Produção. P. Alegre: Bookman, 2001.

DORNIER, P.; ERNST, R.; FENDER, M.; KOUVELIS, P. Logística e operações globais: texto e casos. S. Paulo: Atlas, 2000.

DYER, J. Remarks on the Analytic Hierarchy Process. Management Science, v. 36, n. 3 , p. $249-258,1990$ a.

DYER, J. A clarification of "Remarks on the Analytic Hierarchy Process". Management Science, v. 36, n. 3, p. 274 $275,1990 \mathrm{~b}$

ELLRAM, L.; CARR, A. Strategic purchasing: a history and review of literature. International Journal of Purchasing and Materials Management, v. 30, n. 2, p. 10-18, 1994

ENSLINN, L.; MONTIBELLER, G. NORONHA, S. Apoio à decisão. Insular: Florianópolis, 2001

GASPARETTO, V. Proposta de uma sistemática para avaliação de desempenho em cadeias de suprimentos. Tese de doutorado. EPS - UFSC, Florianópolis, 2003.
GOMES, L; ARAYA, M.; CARIGNANO, C Tomada de decisão em cenários complexos. S. Paulo: Thomson Learning, 2004.

GONÇALVES, P. Administração de materiais. R. Janeiro: Campus, 2004

GUNASEKARAN, A.; PATEL, C.; TIRTIROGLU, E. Performance measures and metrics in a supply chain environment. International Journal of Operations $\&$ Production Management, v. 21, n. 1/2, p. $71-87,2001$

HAIR, J: TATHAM, R.; ANDERSON, R.; BLACK, W. Multivariate data analysis. New Jersey: Prentice Hall, 1998.

HANSEN, P. Um modelo de avaliação de desempenho competitivo de cadeias produtivas. Tese de doutorado. PPGEP-UFRGS, Porto Alegre, 2004.

HARKER, P.; VARGAS, L. Reply "Remarks on the Analytic Hierarchy Process" by J. S. Dyer. Management Science, v. 36, n. 3, p. 269-273, 1990.

HILL, T. Manufacturing strategy: text and cases. London: Macmillian Business, 1995.

KLIPPEL, M.; ANTUNES JR., J. Matriz de posicionamento estratégico dos materiais: uma abordagem metodológica. Anais do XXII ENEGEP, Curitiba, 2002.

KRAUSE, D.; SCANELL, T.; CALANTONE, R. A structural analysis of the effectiveness of buying firms' strategies to improve supplier performance. Decision Sciences, v. 31 , n. 1 , p. $33-55,2000$

KRAUSE, D.; PAGELL, M.; CURKOVIC, S Toward a measure of competitive priorities for purchasing. Journal of Operation Management, n. 19, p. 497-512, 2001.

LAKATOS, E.; MARCONI, M. Metodologia científica. S. Paulo: Ed. Atlas, 1991.

LIU, F; HAI, H. The voting analytic hierarchy process method for selecting supplier. International Journal of Production Economics, n. 97, p. 308-317, 2005.

LOWSON, R. Operations strategy: genealogy, classification and anatomy. International Journal of Operations and Production Management, v. 22, n. 10, p. 1112-1129, 2002

MACHADO, E.; GOMES, L.; CHAUVEL, M Avaliação de estratégias em marketing de serviços: um enfoque multicritério. Revista de Administração Mackenzie, v. 4 n. 2, p. 61-85, 2003.

MALHOTRA, N. Pesquisa de Marketing: uma orientação aplicada. P. Alegre: Artmed, 1999.

MELLO, J.; GOMES, E.; LINS, M. Análise multicritério da presença da Universidade Federal Fluminense com o uso do método MACBETH. Produção, v. 11, n. 2, p. 53-67, 2002. 


\section{- Referências Bibliográficas}

MERLI, G. A nova estratégia de suprimentos. R. Janeiro: Qualitymark, 1994

NEELY, A.; GREGORY, M.; PLATTS, K. Performance measurement system design: A literature review and research agenda. International Journal of Operations and Production Management, v. 15 , n. 4 , p. $80-116,1995$

PAIVA, E.; CARVALHO, L; FENSTERSEIFER, J. Estratégia de produção e de operações. P. Alegre: Bookman, 2004.

PARTOVI, F.; WHITERS, B.; BRADFORD, J. How Tompkins rubber company used Analytic Hierarchy Process to enhance ISO-9000 related decision making. Production and Inventory Management Journal, v. 43, n. 1/2, p. 13-22, 2002.
PEREIRA, J. Análise de dados qualitativos. S. Paulo: Edusp, 1999.

PIRES, S.; ARAVECHIA, C. Measuring supply-chain performance. Anais da XII annual conference of POMS, Orlando, 2001.

PIRES, S. Gestão da cadeia de suprimentos (supply-chain management): conceitos, estratégias, práticas e casos. S. Paulo: Atlas, 2004.

RIBEIRO, J.; NEWMANN, C. Planejamento e condução de grupos focados. In RIBEIRO, J. (org.) Grupos focados: teoria e aplicações. P. Alegre: FEENG-UFRGS PPGEP, 2003.

SAATY, T. An exposition of the AHP in reply to the paper "Remarks on the Analytic
Hierarchy Process". Management Science, v. 36, n. 3, p. 259-267, 1990.

SAATY, T. Método de Análise Hierárquica. S. Paulo: McGraw-Hill, 1991.

SELLITTO, M.; RIBEIRO, J. Construção de indicadores para avaliação de conceitos intangíveis em sistemas produtivos. Gestão \& Produção, v. 11, n. 1, p. 7590,2004

SELLITTO, M. Medição e controle de desempenho estratégico em sistemas de manufatura. Tese (Doutorado em Engenharia de Produção). Escola de Engenharia, UFRGS, P. Alegre, 2005 .

SELLITTO, M.; WALTER, C. Avaliação do desempenho de uma manufatura de equipamentos eletrônicos segundo critérios de competição. Produção, v. 16, n. 1, p. 34-47, 2006.

SIMCH-LEVI, D.; KAMINSKY, P.; SIMCHLEVI, E. Cadeia de suprimentos: projeto e gestão. P. Alegre: Bookman, 2003.

SKINNER, W. Manufacturing - missing link in corporate strategy, Harvard Business Review, may-june, 1969.

SLACK, N. Vantagem competitiva em manufatura. S. Paulo: Atlas, 2002.

WATTS, C.; KIM, Y.; HAHN, C. Linking purchase to corporate competitive strategy. International Journal of Purchasing and Materials Management, v. 28, n. 4 p. 2-8, 1992

\section{- Sobre o autor}

\section{Everton Peter Santos da Rosa}

Universidade do Vale do Rio dos Sinos - Unisinos

Engenheiro de Produção

End.: Rua Marcos Weinstein, 447 - 94930-360 - Cachoeirinha - RS

Tel.: (51) 9288.0306

E-mail: everton.peter@br.elster.com

\section{Miguel Afonso Sellitto}

Universidade do Vale do Rio dos Sinos - Unisinos

Professor e Pesquisador do Programa de Pós-Graduação em Engenharia de Produção e Sistemas

End.: Av. Unisinos, 950 - 93022-000 - São Leopoldo - RS

Tel.: (51) 3591.1122

E-mail: sellitto@unisinos.br

\section{Lia Weber Mendes}

Universidade do Vale do Rio dos Sinos - Unisinos

Professora do curso de Administração de Empresas e professora e coordenadora do MBA UNISINOS em Produção e Logística

End.: Av. Unisinos, 950 - 93022-000 - São Leopoldo - RS

Tel.: (51) 3591.1122

E-mail: LWeber@unisinos.br 